\title{
Limiting saddlepoint relative errors in large deviation regions under purely Tauberian conditions
}

\author{
RONALD W. BUTLER ${ }^{1, *}$ and ANDREW T.A. WOOD ${ }^{2, * *}$ \\ ${ }^{1}$ Department of Statstical Science, Southern Methodist University, Dallas TX 75275 USA \\ E-mail: ${ }^{*}$ rbutler@smu.edu \\ ${ }^{2}$ School of Mathematical Sciences, University of Nottingham, Nottingham NG7 2RD UK \\ E-mail: ${ }^{* *}$ Andrew.Wood@nottingham.ac.uk
}

\begin{abstract}
Most theoretical results on the relative errors of saddlepoint approximations in the extreme tails have involved placing conditions on the density/mass function. Checking the validity of such conditions is problematic when density/mass functions are intractable, as is typically the case in important practical applications involving convolved, compound, and first-passage distributions as well as for moment generating functions MGFs that are regularly varying. In this paper we present novel conditions which ensure the existence of positive finite limiting relative errors for saddlepoint density/mass function and survival function approximations. These conditions, which are rather weak, are expressed entirely in terms of the MGF, hence the description purely Tauberian. We focus mainly on the cases in which there are positive and negative gamma distributional limits (the only other non-degenerate possibility being a Gaussian limit) and we show how to check the new conditions in important classes of models in these two settings.
\end{abstract}

Keywords: saddlepoint approximation, Tauberian arguments, regular variation, first-passage distribution, compound distrbution.

\section{Introduction}

Suppose a random variable $X$ has a moment generating function (MGF) $\mathcal{M}(s)=E\left(e^{s X}\right)$ whose domain of convergence is $\mathcal{S}=\{s \in \mathbb{R}: a \leqq s<b\}$ with $-\infty \leq a \leq 0<b \leq \infty$, where symbol $\leqq$ stands for either $<$ or $\leq$. Saddlepoint approximations are expressed most conveniently in terms of the cumulant generating function (CGF) $\mathcal{K}(s)=\ln \mathcal{M}(s)$ and its derivatives.

Let an absolutely continuous $X$ have density $f(t)$ and survival function $S(t)=1-F(t)$. Define the quantities

$$
\hat{w}=\operatorname{sgn}(\hat{s}) \sqrt{2\{\hat{s} t-\mathcal{K}(\hat{s})\}} \quad \text { and } \quad \hat{u}=\hat{s} \sqrt{\mathcal{K}^{\prime \prime}(\hat{s})},
$$

where the saddlepoint $\hat{s} \in(a, b)$ solves saddlepoint equation $\mathcal{K}^{\prime}(\hat{s})=t$ with $t \in\left(t_{L}, t_{U}\right)$. The interval $\left(t_{L}, t_{U}\right)$ is the subset of $(-\infty, \infty)$ on which there is a unique solution to 
the saddlepoint equation and it is characterized as the interior of the convex hull of the range of $X$. The saddlepoint density approximation is given by

$$
\hat{f}(t)=\frac{1}{\sqrt{2 \pi \mathcal{K}^{\prime \prime}(\hat{s})}} \exp \{\mathcal{K}(\hat{s})-\hat{s} t\}=\frac{\phi(\hat{w})}{\sqrt{\mathcal{K}^{\prime \prime}(\hat{s})}},
$$

where in the right-hand expression $\phi$ is the standard normal density and $\hat{w}$ is defined in (1.1). The Lugannani and Rice (1980) approximation to the survival function $S(t)$ is

$$
\hat{S}(t)=1-\Phi(\hat{w})-\phi(\hat{w})\left(\frac{1}{\hat{w}}-\frac{1}{\hat{u}}\right),
$$

where $\Phi$ is the standard normal cumulative distribution function (CDF), and $\hat{w}$ and $\hat{u}$ are given in (1.1).

Saddlepoint approximations are known to be highly accurate numerically and to have excellent theoretical properties in a wide variety of examples; see for example the books by Jensen (1995) and Butler (2007) for further details.

In this paper we focus on the theoretical relative errors in the extreme tails: in particular, we provide various conditions for the following limits to exist:

$$
\lim _{t \uparrow t_{U}} \frac{f(t)}{\hat{f}(t)} \quad \text { and } \quad \lim _{t \uparrow t_{U}} \frac{S(t)}{\hat{S}(t)} .
$$

We also cover the case where $X$ is lattice- or integer-valued. Under the very weak conditions we propose, the two limits in (1.4) turn out to be the same and the common value is either 1 or $\hat{\Gamma}(\alpha) / \Gamma(\alpha)$ for some $\alpha>0$, where $\Gamma(\alpha)$ is the gamma function and

$$
\hat{\Gamma}(\alpha)=\sqrt{2 \pi} \alpha^{\alpha-1 / 2} e^{-\alpha}
$$

is Stirling's approximation for $\Gamma(\alpha)$. Note that there is no loss of generality in focusing on the right tail in (1.4); for the left tail of $X$, we just focus on the right tail of $-X$.

The study of limiting relative errors of saddlepoint density approximations goes back to the earliest work on saddlepoint approximations in applied probability and statistics; see the landmark paper by Daniels (1954). Daniels (1954) considered four classes of examples under the asymptotic regime indicated in (1.4) and proved that in each class of examples the limiting relative error converged to a positive finite limit. Since the original work of Daniels (1954), the most substantial and extensive work on relative errors of saddlepoint approximations is due to Jensen $(1988,1991 \mathrm{a}, 1991 \mathrm{~b})$, summarized in Chapter 6 of the book Jensen (1995). Other work on relative errors of saddlepoint approximation includes Barndorff-Nielsen and Klüppelberg $(1992,1999)$, who give conditions on the density for the relative error of the saddlepoint approximation to go to zero in the extreme tail, in the univariate and multivariate cases, respectively. They note that the multivariate case is much more challenging.

This paper was motivated principally by the desire to study limits of the type (1.4) in various classes of models, of which the following two classes are the most important: 
(i) first-passage distributions in semi-Markov processes, which are of key importance in electrical engineering, reliability theory and multi-state survival analysis, with the first-passage distribution representing the failure time of a stochastic system or the survival time of a random patient modelled by the process;

(ii) compound distributions, which are of great importance in many areas, especially queueing theory and insurance.

However, the conditions formulated in all previous work on relative errors of saddlepoint approximations are not able to cover most of the interesting cases in (i) and (ii). Indeed, Jensen (1995, ex. 8.2.3, p. 237) provides an example which illustrates some of the technical difficulties that arise in this type of problem.

In this paper we formulate conditions of a completely novel character which enable the development of a comprehensive theory of saddlepoint relative errors in models of type (i) and (ii), along with some other types of models, assuming the relevant MGF exists. See in particular condition (4.2) and Theorem 3. Moreover, we provide auxiliary results which facilitate the checking of condition (4.2) in models of type (i) and (ii), and other classes of models; see Corollaries $1-4$. We also provide other results in the paper including parallel developments for continuous distributions whose support has a finite end-point and for lattice distributions.

A novel feature of most of the results in this paper is that they are purely Tauberian in character; in other words they only require conditions that are expressed entirely in terms of the MGF. [See Korevaar (2004) for a treatise on Tauberian theory.] This is in contrast to nearly all theoretical results in the saddlepoint literature on limiting relative errors, where some conditions have been imposed on the density. We say purely Tauberian rather than Tauberian as the latter terminology has traditionally not excluded placing some quite restrictive assumptions on a density or mass function about which we are trying the draw some conclusions. For example, when using "Tauberian" theorems 4 and 5 of Feller (1971, §XIII.5), in order to reach conclusions about the exponential/geometric decay rate to 0 for the tail of a density and mass function, one must assume that the density/mass function itself is ultimately decreasing in its tail.

The work of Balkema et al. (1999a,b, 2003), who identify what distributional limits can arise for the standardized tilted distribution as $s \uparrow b$ (see $\S 2$ for definitions), has provided a helpful starting point for the work of this paper. They give a number of useful necessary and sufficient conditions for these limits to occur that we have integrated into our development.

Before moving on, we offer a few words on notation and assumptions. Throughout, we deal with regular exponential families in the sense that $\mathcal{S}=\{s \in \mathbb{R}: \mathcal{M}(s)<$ $\infty\}$ is an interval $(a, b)$ or $[a, b)$ and does not include $b$, i.e. $b \notin \mathcal{S}$. Regularity of the exponential family at $b$ implies steepness in the right tail, which in turn guarantees that the saddlepoint equation $\mathcal{K}^{\prime}(\hat{s})=t$ has a unique solution $\hat{s} \equiv \hat{s}(t) \in(0, b)$ for all $t \in$ 
$\left(\mu, t_{U}\right)$ where $\mu=E(X)$; see e.g. Barndorff-Nielsen (1978) for background on exponential families. Whenever "hats" are used, we should think of the relevant quantities as being functions of $t$; when we view quantities as being functions of $s$, as in $t \equiv t(s)=\mathcal{K}^{\prime}(s)$, then hats are not used. For many purposes in this paper it will be equivalent to work with functions of $t$ and functions of $s$ and we shall switch from time to time when convenient. Throughout the paper, the variables $s, t, u$ and $y$ will be real and $z$ and $\omega$ will be complex. When we wish to consider the MGF as a function of a real variable, we shall write $\mathcal{M}(s)$, and when we write $\mathcal{M}(z)$, it means the MGF is being considered as a function of a complex variable; this convention will be helpful to us. Proofs of all theorems and corollaries are given in the appendix in the Supplementary Material, along with some further auxiliary results and additional examples.

\section{Domains of attraction for tilted distributions}

Define $X_{s}$ for $s \in(0, b)$ to be a random variable associated with the $s$-tilted distribution of $X$. For $X$ absolutely continuous with density $f, X_{s}$ has density $f_{s}(t)=$ $e^{s t} f(t) / \mathcal{M}(s)$, and for integer-valued $X$ with mass function $p(n), X_{s}$ has mass function $p_{s}(n)=e^{s n} p(n) / \mathcal{M}(s)$. In either case, let $Z_{s}=\left(X_{s}-\mu_{s}\right) / \sigma_{s}$ denote the standardized tilted variable with $\mu_{s}=\mathcal{K}^{\prime}(s)=E\left(X_{s}\right)$ and $\sigma_{s}=\sqrt{\mathcal{K}^{\prime \prime}(s)}=\sqrt{\operatorname{Var}\left(X_{s}\right)}$, where $\mathcal{K}$ is the cumulant generating function of $X$.

The weak convergence of $Z_{s}$ to $Z$ (denoted $Z_{s} \stackrel{w}{\rightarrow} Z$ ) as $s \uparrow b$ was studied and characterized in Balkema et al. (1999a,b, 2003) when $\mathcal{S}$ does not include its least upper bound $b$ and $Z$ has a non-degenerate distribution. We shall see in $\S 3$ that the limiting relative error ratios in (1.4) for saddlepoint procedures are intimately connected to this weak convergence and to the distribution of $Z$. Generally, the weak convergence $Z_{s} \stackrel{w}{\rightarrow} Z$ as $s \uparrow b$ does not imply convergence of the corresponding MGFs. However, it does in the present context, as was shown by Balkema et al. (1999a, Theorem 3.6). Thus, $Z_{s} \stackrel{w}{\rightarrow} Z$ if and only if the MGF function of $Z_{s}$ converges pointwise to $\mathcal{M}_{Z}(u)$, the MGF function of $Z$, in its convergence region $\left\{u \in \mathbb{R}: \mathcal{M}_{Z}(u)<\infty\right\}$, i.e. for each fixed $u$,

$$
\frac{\mathcal{M}\left(s+u / \sigma_{s}\right)}{\mathcal{M}(s)} \exp \left(-\mu_{s} u / \sigma_{s}\right) \rightarrow \mathcal{M}_{Z}(u) \quad s \uparrow b .
$$

When $b \notin \mathcal{S}$, Balkema et al. (2003) showed that $Z$ can only assume one of the following three distributions: a standard normal $\mathcal{N}$, a standardized gamma $\mathcal{G}(\alpha, 1)$, or a standardized minus gamma $-\mathcal{G}(\alpha, 1)$ distribution. If $Z$ has a standardized gamma $\mathcal{G}(\alpha, 1)$ distribution, then we say that (the right tail of) $X$ is in the domain of attraction of $\mathcal{G}_{\alpha}$ and write $X \in \mathfrak{D}\left(\mathcal{G}_{\alpha}\right)$. Likewise, we define $X \in \mathfrak{D}\left(-\mathcal{G}_{\alpha}\right)$ and $X \in \mathfrak{D}(\mathcal{N})$. Proposition 1 below specifies necessary and sufficient conditions for weak convergence $Z_{s} \stackrel{w}{\rightarrow} Z$ to occur and, in addition, determines the limiting distribution in both the absolutely continuous and integer-valued settings. 
The theory of regular variation also plays an important role in characterizing the weak limit. A function $h: \mathbb{R} \rightarrow \mathbb{R}$ that varies regularly at $s \in \mathbb{R} \cup\{ \pm \infty\}$, with index $\alpha$ satisfying $0 \neq \alpha \in \mathbb{R}$, is said to belong to the class $\mathcal{R} \mathcal{V}_{s}(\alpha)$; to indicate this we sometimes write $h \in \mathcal{R} \mathcal{V}_{s}(\alpha)$. For $s<\infty$, this means that $h(s-t x) / h(s-t) \rightarrow x^{\alpha}$ as $t \downarrow 0$ for all $x>0$, and for $s=\infty$, this means that $h(t x) / h(t) \rightarrow x^{\alpha}$ as $t \uparrow \infty$ for all $x>0$. If either assertion holds with $\alpha=0$, resulting in a limit of 1 , then we say that $h$ belongs to $\mathcal{S} \mathcal{V}_{s}$, the class of functions which are slowly varying at $s$, and sometimes we denote this by $h \in \mathcal{S} \mathcal{V}_{s}$.

Proposition 1 characterizes the settings in which weak convergence can occur (finite limit $\beta$ ) and cannot occur (infinite limit $\beta=\infty$ or no limit).

Proposition 1. (Domains of convergence). Suppose $b \notin \mathcal{S}$, where $b$ and $\mathcal{S}$ are defined $\S 1$.

(a) $Z_{s} \stackrel{w}{\rightarrow} Z$ as $s \uparrow b \leq \infty$ for some random variable $Z$ if an only if $E\left(Z_{s}^{3}\right) \rightarrow \beta$ for some $\beta \in(-\infty, \infty)$. If $Z_{s} \stackrel{w}{\rightarrow} Z$ then

$$
\begin{array}{rlrl}
X \in \mathfrak{D}\left(\mathcal{G}_{\alpha}\right) & \text { if and only if } & & \beta=2 / \sqrt{\alpha}>0 \\
X \in \mathfrak{D}(\mathcal{N}) & \text { if and only if } & \beta=0 \\
X \in \mathfrak{D}\left(-\mathcal{G}_{\alpha}\right) & \text { if and only if } & \beta=-2 / \sqrt{\alpha}<0 .
\end{array}
$$

(b) If $b<\infty$, then the following three statements are equivalent as $s \uparrow b$ :

$$
\text { (i) } X \in \mathfrak{D}\left(\mathcal{G}_{\alpha}\right) ; \quad(\text { ii }) \quad(b-s) \mu_{s} \rightarrow \alpha \in(0, \infty) ; \quad \text { (iii) } \quad \mathcal{M}(s) \in \mathcal{R} \mathcal{V}_{b}(-\alpha)
$$

(c) If $t_{U}<\infty$, then the following three statements are equivalent as $s \uparrow b$ :

(i) $\quad X \in \mathfrak{D}\left(-\mathcal{G}_{\alpha}\right) ; \quad($ ii $) \quad s\left(t_{U}-\mu_{s}\right) \rightarrow \alpha \in(0, \infty) ; \quad$ (iii) $\quad e^{-s t_{U}} \mathcal{M}(s) \in \mathcal{R} \mathcal{V}_{\infty}(-\alpha)$

Parts (a)-(c) are given in Theorems 3.1, 4.3, and 4.7 of Balkema et al. (2003) respectively.

From the proposition, it is apparent that the finite/infinite dichotomies for $b$ and $t_{U}$ determine which of the three domains of convergence are possible. Proposition 2 in $\S 7.1 .1$ of Supplementary Materials provides a detailed account of which limits are possible under this cross classification. Which domain of convergence can occur when approaching $b$ is also related to what sort of singularity type $\mathcal{M}(z)$ has at $b$ when viewed as a function of complex variable $z$. The singularity can be a pole, algebraic or logarithmic branch point, or an essential singularity and $\S 7.1 .2$ of Supplementary Materials details the possibilities for each type of singularity.

\section{Limiting saddlepoint error ratios}

Here we focus on the absolutely continuous setting and consider lattice/integer-valued mass and survival functions in $\S 6$. First we consider limiting ratios for the saddlepoint 
survival function. Theorem 1 below obviates the need to consider the survival ratio $S(t) / \hat{S}(t)$ separately in the continuous case; under the conditions of the theorem it has the same limiting behavior as the density ratio $f(t) / \hat{f}(t)$ as $t \uparrow t_{U}$. Recall the definitions of $\hat{f}(t)$ and $\hat{S}(t)$ in (1.2) and (1.3).

Theorem 1. (Limiting saddlepoint survival ratios). Suppose $b \notin \mathcal{S}$ and

$$
\lim _{t \uparrow t_{U}} \frac{f(t)}{\hat{f}(t)} \in(0, \infty)
$$

exists (e.g. see Theorems 2 and 3).

(a) If $X \in \mathfrak{D}\left(\mathcal{G}_{\alpha}\right)$ or $X \in \mathfrak{D}\left(-\mathcal{G}_{\alpha}\right)$ then

$$
\lim _{t \uparrow t_{U}} \frac{S(t)}{\hat{S}(t)}=\lim _{t \uparrow t_{U}} \frac{f(t)}{\hat{f}(t)} .
$$

(b) If $X \in \mathfrak{D}(\mathcal{N})$ and $\hat{u} / \hat{w}^{3} \rightarrow 0$ as $\hat{s} \rightarrow b$ (see (1.1) for definitions of $\hat{w}$ and $\hat{u}$ ), then (3.1) holds.

The proof of Theorem 1 makes use of the limiting properties for $\hat{w}, \hat{u}$, and $\hat{u} / \hat{w}^{3}$ as $t \uparrow t_{U}$ or equivalently as $s \uparrow b$; see Proposition 5 of $\S 7.1 .3$ of Supplementary Materials for the details. Proposition 5(c) shows that $\hat{u} / \hat{w}^{3} \rightarrow 0$ as $\hat{s} \rightarrow b$ when it is assumed that $X \in \mathfrak{D}\left(\mathcal{G}_{\alpha}\right)$ or $X \in \mathfrak{D}\left(-\mathcal{G}_{\alpha}\right)$. This is the reason that this assumption is not needed in part (a) of Theorem 1 and, accordingly, the conditions for part (a) are very mild. In part (b), the assumption of this condition does not appear restrictive since it holds in the great majority of known cases where $X \in \mathfrak{D}(\mathcal{N})$. It fails in the case of the right tail of the inverse Gaussian distribution, but in this case $b \in \mathcal{S}$ and none of the theory applies. We do not know of any cases where $b \notin \mathcal{S}$ but $\hat{u} / \hat{w}^{3} \rightarrow 0$ fails.

In Theorem 2 below, we assume weak convergence of the standardized tilted random variable $Z_{s}$ as $s \uparrow b$, as well as the existence of a dominating function, plus a very basic assumption on the density $f$ which guarantees that the inversion formula applies. In Theorems 3 and 4 below, where $X \in \mathfrak{D}\left(\mathcal{G}_{\alpha}\right)$ and $X \in \mathfrak{D}\left(-\mathcal{G}_{\alpha}\right)$ respectively, we prove the same result under substantially weaker new conditions which are also easier to check.

Theorem 2. (Limiting saddlepoint error ratios). Suppose $b \notin \mathcal{S}$ and the following two conditions hold:

(i) A dominating function $D$ exists such that, for $b^{-}=b-\varepsilon$ for some $\varepsilon>0$,

$$
\sup _{s \in\left[b^{-}, b\right)}\left|\frac{\mathcal{M}\left(s+i y / \sigma_{s}\right)}{\mathcal{M}(s)}\right| \leq D(y) \quad \forall y \in \mathbb{R} \quad \text { with } \quad \int_{-\infty}^{+\infty} D(y) d y<\infty,
$$


where $\sigma_{s}=\sqrt{\mathcal{K}^{\prime \prime}(s)}=\sqrt{\operatorname{Var}\left(X_{s}\right)}$ (see $\S 2$ ). (ii) $f(t)$ is locally of bounded variation and continuous for sufficiently large $t$.

Then the limiting density ratio is

$$
\lim _{t \uparrow t_{U}} \frac{f(t)}{\hat{f}(t)}=\left\{\begin{array}{clll}
\hat{\Gamma}(\alpha) / \Gamma(\alpha) & \text { if } & X \in \mathfrak{D}\left(\mathcal{G}_{\alpha}\right) & \text { or } \quad X \in \mathfrak{D}\left(-\mathcal{G}_{\alpha}\right) \\
1 & \text { if } & X \in \mathfrak{D}(\mathcal{N}), &
\end{array}\right.
$$

where $\hat{\Gamma}(\alpha)$, defined in (1.5), is Stirling's approximation to $\Gamma(\alpha)$.

The role of $b^{-}$in (3.2) is simply to ensure that we only need to consider an interval of $s$ values which is bounded on the left. This is sometimes helpful when seeking a dominating function. The usefulness of Theorem 2 hinges on whether a dominating function $D$ satisfying (3.2) can be found. There are many important examples where, even though (3.3) holds, a suitable dominating function cannot be found and may not exist.

\subsection{Saddlepoint error ratios with no limit}

In $\S 7.7$ of Supplementary Materials, two continuous examples are provided in which standardized tilted distributions do not converge weakly as $s \uparrow b$. In Example 6 of $\S 7.7$, the lack of convergence can be attributed to $b$ being a logarithmic branch point. Subject to additional assumptions, the saddlepoint relative errors are not bounded as $s \uparrow b$.

In the second of these examples, Example 9 of $\S 7.7$, the density has a dampened oscillatory form and there is again no weak convergence. However the saddlepoint relative errors remain bounded as $s \uparrow b$ but do not converge and are ultimately oscillatory.

\section{The case $X \in \mathfrak{D}\left(\mathcal{G}_{\alpha}\right)$}

Assume that

$$
\mathcal{M}(z)=(b-z)^{-\alpha} g(z)
$$

for $\alpha>0$ where $g$ is analytic on $\{z \in \mathbb{C}: 0<\operatorname{Re}(z)<b\}$ and $(b-z)^{-\alpha}$ assumes principal values using a branch cut on $[b, \infty]$ if $\alpha$ is not a positive integer. Note that $g$ need not be proportional to an MGF. Indeed, in the most interesting classes of examples considered in $\S 4.1, g$ will usually not be proportional to an MGF. In the case that $\alpha$ is integer-valued so $b$ is a pole, then $g(z)=(b-z)^{\alpha} \mathcal{M}(z)$ represents a function without a singularity at $b$ so that often $g$ may be analytically continued to the right of $b$.

Theorem 3. $\left(X \in \mathfrak{D}\left(\mathcal{G}_{\alpha}\right)\right)$. For an absolutely continuous distribution with moment generating function $\mathcal{M}$ of the form (4.1), suppose $X \in \mathfrak{D}\left(\mathcal{G}_{\alpha}\right)$ and that the following condition 
holds for either $j=1$ or $j=2$ : for some $\varepsilon>0, g$ in (4.1) satisfies

$$
\sup _{s \in\left[b^{-}, b\right)}\left|\frac{1}{\sigma_{s}^{j}} \frac{g^{(j)}\left(s+i y / \sigma_{s}\right)}{g(s)}\right| \leq c_{j}(1+|y|)^{\alpha-1-\varepsilon}
$$

where $g^{(j)}$ denotes the $j^{\text {th }}$ complex derivative of $g, b^{-} \in(0, b)$ is arbitrary but fixed, $\sigma_{s}$ is defined as in Theorem 2 and $c_{j}$ is a constant. Then

$$
\lim _{t \uparrow t_{U}} \frac{f(t)}{\hat{f}(t)}=\lim _{t \uparrow t_{U}} \frac{S(t)}{\hat{S}(t)}=\frac{\hat{\Gamma}(\alpha)}{\Gamma(\alpha)} .
$$

In (4.2), any choice $b^{-} \in(0, b)$ will do. When applying the theorem, it is best to try to check condition (4.2) with $j=1$ first, and if it fails to hold with $j=1$, then try to check condition (4.2) with $j=2$.

Apart from the assumption of absolute continuity, no assumptions are made in Theorem 3 about the density function $f$ or survival function $S$. The avoidance of assumptions on $f$ plus the novel nature of condition (4.2) sets Theorem 3 apart from all previous results on saddlepoint relative errors in the literature; cf. Daniels (1954) and Jensen (1995). The assumptions which are made, that the MGF takes the form (4.1) and satisfies (4.2) with either $j=1$ or $j=2$, are expressed entirely in terms of the MGF $\mathcal{M}$.

The condition $X \in \mathfrak{D}\left(\mathcal{G}_{\alpha}\right)$ can also be based entirely on the form of $\mathcal{M}$. From part (b) of Proposition 1, it follows that $g(s)$, when viewed as a function of real variable $s$, must be slowly varying as $s \uparrow b$ and $\mathcal{M}$ must be regularly varying at $b$. This holds if $g(z)$ is analytic at $z=b$ and is the case in many practical settings such as when $b$ is an isolated pole for $\mathcal{M}$. For $g$ not easily recognizable as slowly varying, then verification follows from part (a) of Proposition 1 if the third standardized tilted moment is shown to have a positive finite limit.

Although condition (4.2) with $j=1$ suffices in nearly all the cases we have seen, it does in fact fail in some cases of Example 1 below. However, in this example (4.2) holds in all cases when $j=2$.

Example 1: (Generalized inverse Gaussian distribution, GIG $(p, \beta, \gamma)$ ). The $\operatorname{GIG}(p, \beta, \gamma)$ has density function and MGF given by, respectively,

$$
f(t)=\frac{(\beta / \gamma)^{p / 2}}{2 K_{p}(\sqrt{\beta \gamma})} t^{p-1} \exp \left(-\frac{\beta t+\gamma / t}{2}\right), \mathcal{M}(s)=\left(\frac{\beta}{\beta-2 s}\right)^{p / 2} \frac{K_{p}(\sqrt{\gamma(\beta-2 s)})}{K_{p}(\sqrt{\gamma \beta})}
$$

where $t>0, K_{p}(\cdot)$ is a modified Bessel function, $p \in \mathbb{R}, \beta>0$ and $\gamma>0$ if $p \leq 0$ and $\gamma \geq 0$ if $p>0$. See Abramowitz and Stegun (1972) for details of $K_{p}$ and Jorgensen (1980) for details of the GIG distribution. The GIG family exhibits some diversity of behavior 
in terms of limiting saddlepoint relative errors. We first focus on the two cases for which the results of this paper are relevant: the right tail when $p>0$ and the left tail when $\gamma>0$ (note that if $\gamma=0$ we must have $p>0$ for the density to be proper, in which case we recover the gamma distribution). We then briefly explain what happens in the third case, the right tail when $p \leq 0$.

When $p>0$, it turns out that the right tail of $\operatorname{GIG}(p ; \beta, \gamma)$ is in $\mathfrak{D}\left(\mathcal{G}_{p}\right)$. However, it is shown in $\S 7.7$ of Supplementary Materials that (4.2) with $j=1$ holds when $p>1 / 2$ and fails when $p \in(0,1 / 2]$, while condition (4.2) with $j=2$ is satisfied for all $p>0$ (with $\alpha=p$ ) so that the limits in (4.3) hold for all $p=\alpha>0$. This is the only example we know of where (4.3) holds but (4.2) with $j=1$ fails to hold.

For the left tail, any member of the GIG family (4.4) with $\gamma>0$ is in $\mathfrak{D}(\mathcal{N})$. It is shown in $\S 7.7$ of Supplementary Materials that condition (3.2) of Theorem 2 holds with dominating function $D(y)=c_{1} \exp \left(-c_{2} y^{1 / 2}\right)$ for some sufficiently large positive constant $c_{1}$ and sufficiently small positive constant $c_{2}$. It can be checked that $\hat{u} / \hat{w}^{3} \rightarrow 0$ as $t \rightarrow 0$ so Theorem 1 also holds. Thus, for any parametrization of the GIG $(p ; \beta, \gamma)$ distribution, the limiting saddlepoint density and survival ratios are 1 as $t \rightarrow 0$. For a GIG $(2 ; 1,1)$ distribution, at $t_{0}=1.11 \times 10^{-8}$ the saddlepoint density ratio is $1-10^{-17}$. For the survival ratio at $t_{1}=0.00319, F\left(t_{1}\right)=1.453 \times 10^{-76}$ and $\hat{F}\left(t_{1}\right)=1.455 \times 10^{-76}$ which gives the ratio 0.9984 . For a GIG $(-2 ; 1,1)$ distribution, at $t_{2}=1.12 \times 10^{-8}$, the saddlepoint density ratio is $1-10^{-17}$ and at $t_{3}=0.00352, F\left(t_{3}\right)=3.165 \times 10^{-60}$ and $\hat{F}\left(t_{3}\right)=3.171 \times 10^{-60}$ for a ratio of 0.9981 .

The third case consists of the right tail when $p \leq 0$. Here, $b \in \mathcal{S}$, so this is outside the domain of the Balkema et al. (1999a, 2003) results. When $p<0$ it turns out that the standardized tilted distribution $Z_{s}$ is degenerate in the limit $s \uparrow b=\beta / 2$. The case $p=0$ is intriguing as it is at the boundary of $p<0$ and $p>0$. As $s \uparrow b$, there is no weak convergence and the saddlepoint density ratio is unbounded as shown in $\S 7.7$ of Supplementary Materials.

There is, however, an interesting twist to the third case. The inverse Gaussian distribution is the special case GIG $(-1 / 2 ; \beta, \gamma)$ and steepness holds at $b=\beta / 2$, despite the fact that $\mathcal{M}(\beta / 2)<\infty$ and the distributional limit of $Z_{s}$ is degenerate. The saddlepoint density for the inverse Gaussian density is exact (Daniels, 1980) and the limiting survival ratio differs by assuming the value 2 rather than 1; see Barndorff-Nielsen (1990, p. 491) and Booth (1994). In the left tail, $X \in \mathfrak{D}(\mathcal{N})$ and the limiting relative CDF error is 1 in agreement with Barndorff-Nielsen (1990, p. 491).

Irregular saddlepoint cases $(p<0)$. Right tail saddlepoint approximations in this setting for which $X \sim \operatorname{GIG}(p ; \beta, \gamma)$ can be realized by working with the left tail of $1 / X \sim \operatorname{GIG}(-p ; \gamma, \beta)$ where $-p>0$ and now $1 / X \in \mathfrak{D}(\mathcal{N})$.

The limiting relative error in (4.3) can also be shown to hold subject to other condi- 
tions. For example, it suffices that $\mathcal{M}(z)$ is complex regularly varying at $b$ for $z \in \mathbb{C}$; see $\S 7.6$ and Corollary 9 .

Our next example has two purposes: first, to exhibit a case where weak convergence of the standardized tilted distribution for $Z_{s}$ occurs as $s \uparrow b$, and the relative error stays bounded, yet the limits (4.3) do not hold; and secondly to show that condition (4.2) of Theorem 3 is strictly weaker than the sufficient conditions of Corollary 9 in (7.91) of $\S 7.6$ which assume $\mathcal{M}(z)$ is complex regularly varying.

Example 2. (Wobbly density). The density $f(t ; \theta)=c(\theta)\{1+\sin (\theta t)\} e^{-t}$, for $t \geq 0$ and $c(\theta)=\left(1+\theta^{2}\right) /\left(1+\theta+\theta^{2}\right)$, has MGF

$$
\mathcal{M}_{\theta}(z)=c(\theta) \frac{(1-z)^{2}+\theta^{2}+\theta(\theta+1-z)}{(1-z)(1+\theta i-z)(1-\theta i-z)}, \quad \operatorname{Re}(z)<1=b,
$$

with simple poles at 1 and $1 \pm \theta i$, which "interfere" with one another to create a wobbly density. The poles at $1 \pm \theta i$ do not affect the domain of attraction, and using Proposition 1 it can be shown that $X \in \mathfrak{D}\left(\mathcal{G}_{1}\right)$ in the right tail and $X \in \mathfrak{D}\left(-\mathcal{G}_{1}\right)$ in the left tail. These poles, however, do affect the relative error; direct computation shows that

$$
\frac{f(t)}{\hat{f}(t)} \sim \frac{1+\sin (\theta t)}{\hat{\Gamma}(1)}, \quad t \rightarrow \infty
$$

and the relative error is ultimately $\theta /(2 \pi)$-periodic, so it is bounded but without a limit.

We now show how the wobbly density can be used to construct a distribution for which (4.2) holds with $j=1$ yet the assumptions of Corollary 9 in (7.91) fail. Let $\mathcal{M}_{\theta}(z)$ be the wobbly density MGF in (4.5), and consider the mixture distribution with MGF

$$
\mathcal{M}(z)=\frac{1}{2} \frac{1}{(1-2 z)}+\frac{1}{2} \sum_{\theta=1}^{\infty}\left(\frac{1}{2}\right)^{\theta} \mathcal{M}_{\theta}(z)
$$

with simple poles at $b=1 / 2$ and at $\{1 \pm \theta i: \theta=0,1, \ldots\}$. It is a simple but tedious exercise to show that this distribution satisfies (4.2) with $j=1$; however, its MGF cannot be in the complex regularly varying class $\mathcal{C R} \mathcal{V}_{b}(-1, \vartheta)$ for some $\vartheta \in(\pi / 2, \pi)$. The infinite sequence of poles along the line $\{\operatorname{Re}(z)=1\}$ prevents $g$ from being analytic on any sector centered at $b=1 / 2$ and including an angular portion of the analytic continuation. Hence, the requirement of Corollary 9 that $\vartheta \in(\pi / 2, \pi)$ fails.

\subsection{Applications for $X \in \mathfrak{D}\left(\mathcal{G}_{\alpha}\right)$}

We now consider four important classes of applications of Theorem 3. The first three classes have a common structure in which $\mathcal{M}(z)$, the MGF of interest, is analytic on $\{0<\operatorname{Re}(z)<b\}$ and may be be expressed as a function of a finite number of input 
MGFs $\left\{\mathcal{M}_{j}\right\}_{j=1}^{p}$, i.e. for a suitable function $\mathcal{F}, \mathcal{M}(z)=\mathcal{F}\left\{\mathcal{M}_{1}(z), \ldots, \mathcal{M}_{p}(z)\right\}$. The following condition will often be placed on input MGFs in order to ensure that (4.2) holds with $j=1$.

For some $\varepsilon>0$, let the moment generating function $\mathcal{M}_{0}(z)$ be analytic on $\{z \in \mathbb{C}: 0<$ $\operatorname{Re}(z)<b+\varepsilon\}$. Also, for some $\delta>0$ and constant $c_{0} \in(0, \infty)$, assume that

$$
\sup _{s \in\left[b^{-}, b\right)}\left|\mathcal{M}_{0}^{\prime}(s+i y)\right| \leq c_{0}(1+|y|)^{-1-\delta},
$$

where $\mathcal{M}_{0}^{\prime}(z)$ is the complex derivative of $\mathcal{M}_{0}(z)$.

In the situations of interest here, $\mathcal{M}_{0}$ will automatically be analytic up to $\operatorname{Re}(z)<b+\varepsilon$ due to the nature of the transformation $\mathcal{F}$, as we shall see below. Almost all of the commonly used families of probability densities which possess the relevant exponential moments satisfy condition (4.6), including for example all gamma distributions, all generalized inverse Gaussian distributions which have some finite exponential moments, finite mixtures of these distribution and infinite mixtures which have some finite exponential moments.

\subsubsection{Class 1: Convolutions of moment generating functions}

Here, the function $g$ in (4.1) is $g(z)=b^{\alpha} \mathcal{M}_{0}(z)$, where $\mathcal{M}_{0}$ is itself an MGF.

Corollary 1. (Convolutions). Suppose $X$ has moment generating function $\mathcal{M}$ as in (4.1) and $g(z)=b^{\alpha} \mathcal{M}_{0}(z)$, where $\mathcal{M}_{0}$ is a moment generating function. If $\mathcal{M}_{0}$ satisfies condition (4.6) then condition (4.2) with $j=1$ holds, $X \in \mathfrak{D}\left(\mathcal{G}_{\alpha}\right)$, and the saddlepoint limits in (4.3) hold.

Corollary 1 is satisfied by members of the Daniels' (1991) class discussed in Jensen $(1995, \S 10.2)$. The class includes the asymptotic null distributions for various normal goodness-of-fit tests including the Kolmogorov-Smirnov test. The class consists of convolutions of independent and non-identically distributed gamma variables $X_{n} \sim$ Gamma $\left(\gamma_{n}, b_{n}\right)$ for which $\sum_{n \geq 1} X_{n}$ converges a.s. and $b_{1}<\inf _{n \geq 2} b_{n}$. The component $X_{1}$ dominates and $\sum_{n \geq 2} X_{n}$ contributes MGF $\mathcal{M}_{0}$. By Corollary 1, the limiting ratios are $\hat{\Gamma}\left(\gamma_{1}\right) / \Gamma\left(\gamma_{1}\right)$.

\subsubsection{Class 2: First-passage moment generating functions in semi-Markov processes}

Semi-Markov processes generalize Markov processes by replacing the exponential and geometric holding time distributions in states with very general distributions. Additionally, and unlike Markov processes, these holding time distributions are allowed to depend on destination states during the holding as, for example, would occur in first-passage 
distributions for a GI/M/1 or M/G/1 queue (Butler, 2007, §13.2.5). In an $m$-state semiMarkov process with state space $\mathfrak{S}=\{1, \ldots, m\}$, the dynamics can be explained as they would for a Markov chain: an $m \times m$ jump chain $\mathbf{P}=\left\{p_{i j}\right\}$ determines 1-step transitions in states and an $m \times m$ matrix of holding time CDFs $\left\{F_{i j}(t)\right\}$ specifies destination dependent holding times. However, rather than working with $\left\{F_{i j}(t)\right\}$, we consider the corresponding matrix of MGFs $\mathbf{M}(z)=\left\{\mathcal{M}_{i j}(z)\right\}$. Together, $\mathbf{P}, \mathbf{M}(z)$, and a starting state characterize the semi-Markov process. Equivalently, the transmittance matrix $\mathbf{T}(s)=\mathbf{P} \odot \mathbf{M}(z)=\left\{p_{i j} \mathcal{M}_{i j}(z)\right\}$ and a starting state characterize the process.

Let $X$ be any first-passage time in such a process which can be specified as first passage from state $1 \rightarrow m \neq 1$ with an appropriate relabelling of the states. The first-passage transmittance $E\left\{e^{z X} 1_{\{X<\infty\}}\right\}$ from $1 \rightarrow m \neq 1$ is given in Butler $(2000, \S 4.1)$ as

$$
\mathcal{F}_{1 m}(z):=E\left\{e^{z X} 1_{\{X<\infty\}}\right\}=\frac{(m, 1) \text { cofactor of } \mathbf{I}_{m}-\mathbf{T}(z)}{(m, m) \text { cofactor of } \mathbf{I}_{m}-\mathbf{T}(z)}=: \frac{(-1)^{m+1}\left|\mathbf{\Psi}_{m 1}(z)\right|}{\left|\mathbf{\Psi}_{m m}(z)\right|}
$$

for $\operatorname{Re}(z)<b$, where $\mathbf{I}_{m}$ is the $m \times m$ identity matrix. If all states in $\mathfrak{S}$ communicate and $\mathcal{M}_{i j}$ has convergence boundary $b_{i j}>0$ for all $i, j \in \mathfrak{S}$, then it can be shown that $0<b<\min _{(i, j) \in \mathfrak{S}_{\backslash m} \times \mathfrak{S}_{\backslash m}} b_{i j}$ where $\mathfrak{S}_{\backslash m}=\mathfrak{S} \backslash\{m\}$; additionally, if we assume $b<\min _{i \in \mathfrak{S}_{\backslash m}} b_{i m}$, then together $b<b_{i j}$ for all the $\mathcal{M}_{i j}$ which appear in the ratio (4.7) (the $m$ th row of $\mathbf{M}(z)$ does not appear in (4.7)). More recently, Butler (2018) has shown that if $\mathfrak{S}$ consists of an irreducible subchain along with all transients states that are possible intermediate states during the sojourn $1 \rightarrow m$, then $b$ is a simple zero of $\left|\Psi_{m m}(z)\right|$ and a dominant pole of the MGF $\mathcal{M}(z)=\mathcal{F}_{1 m}(z) / \mathcal{F}_{1 m}(0)$. By dominant, we mean no other singularities exist on the boundary $\{z \in \mathbb{C}: \operatorname{Re}(z)=b\}$. In this context,

$$
g(z)=\frac{(-1)^{m+1}\left|\boldsymbol{\Psi}_{m 1}(z)\right|}{\mathcal{F}_{1 m}(0)} \frac{b-z}{\left|\Psi_{m m}(z)\right|}
$$

where $g(z)$ has a removable singularity at $z=b$.

Our main result in this subsection can now be stated for limiting saddlepoint ratios related to approximating the first-passage density and survival functions.

Corollary 2. (First-passage distributions). For a semi-Markov process as described above, suppose a first-passage distribution has moment generating function given in (4.7). If $\mathcal{M}_{i j}(z)$ satisfies condition (4.6) for all $(i, j) \in \mathfrak{S}_{\backslash m} \times \mathfrak{S}$, then $g$ in (4.8) satisfies condition (4.2) with $j=1$ and therefore the limiting saddlepoint ratios are given in (4.3) as $\hat{\Gamma}(1)$, where $\hat{\Gamma}(\cdot)$ is defined in (1.5).

\subsubsection{Class 3: Compound distributions}

Suppose $X_{1}, X_{2}, \ldots$ is an independent and identically distributed sequence and, independent of the sequence, $N$ is a positive integer-valued random variable. The focus of our 
interest in this subsection is the random sum $S_{N}=\sum_{i=1}^{N} X_{i}$ with a compound distribution. Suppose $X_{1}$ has density $f_{0}$ and MGF $\mathcal{M}_{0}(z), N$ has probabilities $\left\{p_{n}: n=1,2, \ldots\right\}$ and for each fixed integer $n \geq 1, f_{0}^{(n)}$ is the density of the sum $X_{1}+\cdots+X_{n}$. Then it follows from elementary considerations that the density of $S_{N}$ and its MGF are

$$
f(t)=\sum_{n=1}^{\infty} p_{n} f_{0}^{(n)}(t), \quad \mathcal{M}(z)=\sum_{n=1}^{\infty} p_{n} \mathcal{M}_{0}^{n}(z)=\mathcal{P}\left\{\mathcal{M}_{0}(z)\right\}
$$

where $\mathcal{P}(\omega)=\sum_{n \geq 1} p_{n} \omega^{n}$ denotes the probability generating function (PGF) for $N$. Our aim now is to find conditions on $\mathcal{M}_{0}$ and $\mathcal{P}$ under which the saddlepoint density and survival function approximations of $f(t)$ and $S(t)$, respectively, satisfy (4.3).

We suppose $\mathcal{P}$ has radius of convergence $r>1$ and has a pole of order $\alpha>0$ at $r$ so $\mathcal{P}(\omega)$ may be written as

$$
\mathcal{P}(\omega)=(r-\omega)^{-\alpha} G(\omega) .
$$

Assume $\mathcal{M}_{0}(z)$ is convergent on at least $\{0<\operatorname{Re}(z)<c\}$ with $r<\mathcal{M}_{0}(c) \leq \infty$. Let $b>0$ be the unique solution to $\mathcal{M}_{0}(s)=r$ with multiplicity 1 (since $\mathcal{M}_{0}(s)$ is strictly increasing in $s \in \mathbb{R})$. We may define $C(z)$ such that $r-\mathcal{M}_{0}(z)=(b-z) C(z)$, in which case

$$
\mathcal{M}(z)=(b-z)^{-\alpha} g(z), \quad g(z)=G\left\{\mathcal{M}_{0}(z)\right\}\{C(z)\}^{-\alpha} .
$$

Our main result for compound MGFs is now stated.

Corollary 3. (Compound distributions). For such a distribution as described above, if $\mathcal{M}_{0}$ satisfies condition (4.6) then condition (4.2) with $j=1$ is satisfied and the limiting saddlepoint ratios are given by (4.3).

Jensen (1995, Chapter 7) also considers relative errors for compound distributions though he takes a somewhat different perspective than that used here. Important subclasses of such compound distributions include the Pollaczek-Khintchine MGF in queuing theory and the Cramér-Lundberg model in insurance mathematics. The latter was also considered in Jensen (1995, Theorem 10.3.2) whose approach to dealing with the relative error of $S(t) / \hat{S}(t)$ is direct in that the Cramér-Lundberg expansion for $S(t)$ is compared to an expansion for $\hat{S}(t)$. By contrast, the approach taken in this paper is indirect as it does not use the Cramér-Lundberg expansion but rather deals with relative error through inversion formulas. The approach used here also provides relative errors for the saddlepoint density ratio, i.e. the first expression in (4.3).

\subsubsection{Class 4: $\mathcal{M}$ varies regularly at $s=b$.}

In this case we assume that $\mathcal{M}$ has the form (4.1) where now

$$
g(z)=b^{\alpha} L\{1 /(b-z)\}
$$


and $L$ is a function which is slowly-varying at infinity. Consider the following condition on $L$ : for some fixed $\varepsilon>0$, the function $L$ satisfies

$$
\sup _{s \in\left[b^{-}, b\right)}\left|\frac{1}{\left(b-s-i y / \sigma_{s}\right)} \frac{L^{\prime}\left\{1 /\left(b-s-i y / \sigma_{s}\right)\right\}}{L\{1 /(b-s)\}}\right|=O\left\{(1+|y|)^{\alpha-\varepsilon}\right\} \quad \text { as } \quad|y| \rightarrow \infty .
$$

From (4.9), the argument of $L^{\prime}$ converges to $0 \in \mathbb{C}$ as $|y| \rightarrow \infty$ so that it is the behavior of $L^{\prime}$ in a neighborhood of $0 \in \mathbb{C}$, rather than the behavior of $L^{\prime}$ at infinity, that is critical.

Corollary 4. $(\mathcal{M}$ varies regularly at $b)$. Suppose $\mathcal{M}$ varies regularly at $b$ as described above and $L$ satisfies condition (4.9). Then, condition (4.2) with $j=1$ is satisfied and the saddlepoint limits in (4.3) hold.

Condition (4.9) is satisfied by a large class of functions $L$ which are unbounded and slowly-varying at infinity, including all powers of logarithms and all powers of iterated logarithms. The importance of condition (4.9) is that it allows a purely Tauberian condition to be specified in order to obtain the saddlepoint limits in (4.3) rather than the mixed conditions (i.e. including an assumption on the density) that would ordinarily be used in the traditional theory of Hardy-Littlewood-Karamata (H-L-K). To conclude the limits in (4.3) for the latter theory, one needs to not only assume that $\mathcal{M}$ varies regularly at $b$, but also that the the $b$-tilted density $e^{b t} f(t)$ (or the appropriately tilted mass function) is ultimately monotone as $t \uparrow t_{U}$. An argument for this is detailed in the next paragraph. In Corollary 4, we have avoided imposing ultimately monotone conditions on the tilted density, which may be very difficult to check in specific cases, and have rather imposed condition (4.9) on the MGF.

We now indicate why (4.3) holds using traditional H-L-K theory under mixed conditions, i.e. that $\mathcal{M}$ varies regularly and $e^{b t} f(t)$ is ultimately monotone as $t \rightarrow \infty$. The H-L-K theory (Feller, 1972, §XIII.5 Theorems 4 and 5; Bingham et al., 1987, Theorem 1.7.2 and Corollary 1.7.3) is applied rather to the improper $b$-tilted density $e^{b t} f(t)$ and not directly to $f(t)$ itself; this approach is described in Butler $(2017, \S 3)$ and is specified under the "Feller conditions". From the H-L-K theory, we obtain an asymptotic expression for $f(t)$ as $t \uparrow t_{U}$. This, along with the regularly varying form for $\mathcal{M}$ and its ensuing saddlepoint properties, allows the limits in (4.3) to then be derived using some straightforward computations.

\section{The case $X \in \mathfrak{D}\left(-\mathcal{G}_{\alpha}\right)$}

The most common setting for this case is the left tail of a distribution for which $Y \geq 0$, in which case $X=-Y$ has upper support bound $t_{U}=0$. We shall continue to work in the right tail with variable $X$ instead of $Y$ and assume without loss in generality that 
$t_{U}=0$. If we are interested in the right tail of $Y$ and it has upper support bound at $t_{U} \in(0, \infty)$, then we take $X=Y-t_{U}$ so we again are working with $t_{U}=0$ for $X$ with MGF $\mathcal{M}(s)$.

Theorem 4. $\left(X \in \mathfrak{D}\left(-\mathcal{G}_{\alpha}\right)\right)$. Suppose $X$ has $t_{U}=0$ and moment generating function $\mathcal{M}(z)$ with the following uniform asymptotic expansion: for some $\alpha>0, \eta>0$ and $\varepsilon>0$,

$$
\mathcal{M}(z)=c_{1} / z^{\alpha}+O\left(1 / z^{\alpha+\eta}\right)
$$

holds uniformly as $|z| \rightarrow \infty$ for $|\arg (z)| \leq \pi / 2-\varepsilon$. Then $X \in \mathfrak{D}\left(-\mathcal{G}_{\alpha}\right)$ and

$$
\lim _{t \uparrow 0} \frac{S(t)}{\hat{S}(t)}=\frac{\hat{\Gamma}(\alpha)}{\Gamma(\alpha)} .
$$

Additionally, suppose $f(t)$, the density of $X$, is continuous and locally of bounded variation for $t \in\left(-\varepsilon_{0}, 0\right)$ for some $\varepsilon_{0}>0$. Then

$$
\lim _{t \uparrow 0} \frac{f(t)}{\hat{f}(t)}=\frac{\hat{\Gamma}(\alpha)}{\Gamma(\alpha)} .
$$

When considering the left tail of $Y \geq 0$ with $\mathrm{CDF} F_{Y}(t)$, the expansion in (5.1) concerns $\mathcal{M}_{Y}(-z)=E\left\{e^{-z Y}\right\}$, the Laplace transform, and the conclusion in (5.2) concerns the distribution function $F_{Y}$ with

$$
\lim _{t \downarrow 0} \frac{F_{Y}(t)}{\hat{F}_{Y}(t)}=\frac{\hat{\Gamma}(\alpha)}{\Gamma(\alpha)}
$$

where $\hat{F}_{Y}(t)=1-\hat{S}_{Y}(t)$ is the saddlepoint approximation for $F_{Y}(t)$.

In the next result, we provide simple conditions on the density $h(t)=f(-t)$ of $-X>0$ which ensure that expansion (5.1) holds for the MGF of $X$. Let $h(z)$ denote the analytic continuation of density $h$ to $\mathbb{C}$ and assume there exists an $\varepsilon_{0}>0$ and $r>0$ such that $h(z)$ may have a branch point at 0 but is otherwise complex analytic on the sector $V_{\varepsilon_{0}}(r)=\left\{z \in \mathbb{C}: 0<|z| \leq r,|\arg (z)| \leq \varepsilon_{0}<\pi\right\}$. Also, for $z \in V_{\varepsilon_{0}}(r)$ and some $\beta \in[0,1)$, assume that

$$
h(z)=z^{-\beta} \sum_{k=0}^{\infty} h_{k} z^{k} \quad 0<|z| \leq r-\varepsilon_{1}
$$

for some $\varepsilon_{1}>0$, where $h_{0} \neq 0$ if $\beta \in(0,1)$.

The condition $\beta \in[0,1)$ is not a restriction as we now explain. If $\beta \in(0,1)$ and $z=0$ is a branch point, then $h_{0} \neq 0$ ensures that $h(t)=O\left(t^{-\beta}\right)$ as $t \downarrow 0$ and $\beta \in(0,1)$ is required for $h$ to be an integrable density on $\left(0, \varepsilon_{2}\right)$ for some $\varepsilon_{2}>0$. Perhaps the most common setting in which (5.4) holds is the setting in which $h(t)=t^{-\beta} d(t) 1_{\{t>0\}}$ or is 
a sum of such terms, where $d(t)$ is real analytic at $t=0$ with an analytic continuation that is complex analytic at $z=0$.

Corollary 5. (Sufficient conditions for (5.1)). Suppose $-X$ has support in $(0, \infty)$. If $h(t)$, the density for $-X$, satisfies the condition in (5.4), then a uniform expansion as in (5.1) exists with $\alpha=m+1-\beta$ where $m=\min \left\{k \geq 0: h_{k} \neq 0, h_{j}=0\right.$ for $\left.j<k\right\}$.

Example 3 (Uniform convolutions). Daniels $(1954, \S 5)$ considered $Y$ as a convolution of $n$ independent Uniform $(0,1)$ variables so $t_{U}=n$. Using direct arguments, he showed that the limiting density ratio for $Y-n$ in (5.3) holds with $\alpha=n$ but did not show this for the survival ratio in (5.2). Both limiting ratios hold since (5.1) holds with $\mathcal{M}(z)=\{(1-$ $\left.\left.e^{-z}\right) / z\right\}^{n} \sim z^{-n}$. The condition (5.4) also holds. From Feller (1971, I.9 Theorem 1) we can determine the convolution density for $-X=n-Y$ in $t \in(0,1)$ is $h(t)=t^{n-1} /(n-1)$ !. Thus in (5.4), $\beta=0$ and $m=n-1$ leading to $\alpha=n$.

Example 4. (Truncated density). For an arbitrary absolutely continuous variable $Z$ with density $f$ on $\mathbb{R}$, let $Y$ be the distribution restricted to $Z>0$ so it has density $f(t) / S(0)$ for $t>0$. Limiting saddlepoint ratios as $t \downarrow 0$ are determined by applying either (5.1) to the MGF of $X=-Y$ or else (5.4) to the density of $Y$. For example, if $Z \sim N(0,1)$ is standard normal, then $X$ is $Z$ conditional on $Z<0$ with MGF

$$
\mathcal{M}(z)=\frac{\Phi(-z)}{2 \sqrt{2 \pi} \phi(-z)}=\frac{\operatorname{erfc}(z / \sqrt{2})}{4 \sqrt{2 \pi} \phi(z)}, \quad z \in \mathbb{C}
$$

where $\operatorname{erfc}(z)=1-\operatorname{erf}(z), \operatorname{erf}(\cdot)$ is the classical error function, and the expression (5.5) is essentially Mill's ratio. Here, $\mathcal{M}$ is an entire function and has an essential singularity at $b=\infty$ since $\mathcal{M}(s) / s^{n} \rightarrow \infty$ as $\mathbb{R} \ni s \rightarrow-\infty$ for any $n$. However, in the other tail, $\mathcal{M}(z)$ has the asymptotic expansion in the complex plane given by

$$
\mathcal{M}(z) \approx \frac{1}{2 \sqrt{2 \pi}} \frac{1}{z} \sum_{n=0}^{\infty} \frac{(-1)^{n}(2 n) !}{2^{n} n ! z^{2 n}}, \quad z \rightarrow \infty,|\arg (z)|<3 \pi / 4
$$

(see Abramowitz and Stegun $(1972,7.1 .23)$ for the expansion of $\operatorname{erfc}(z / \sqrt{2}) / \phi(z)$ ), so (5.1) holds and $X=-Y \in \mathfrak{D}\left(-\mathcal{G}_{1}\right)$ as $t \downarrow 0$.

This example illustrates a common setting in which $X \in \mathfrak{D}\left(-\mathcal{G}_{\alpha}\right)$ as $t \uparrow 0$. The MGF $\mathcal{M}(z)$ of $X$ is entire and not a finite polynomial so it has an essential singularity at $\infty$. The Taylor expansion of $\mathcal{M}(z)$ holds on $\mathbb{C}$ but a different divergent expansion as in (5.6) holds in a sector including $\{z \in \mathbb{C} ; \operatorname{Re}(z) \geq 0\}$ such as $\{z \in \mathbb{C}:|\arg (z)|<3 \pi / 4\}$ for Example 4. The setting is an expression of Stokes phenomenon in which different asymptotic regimes for $\mathcal{M}(z)$ hold in different sectors with the Stokes lines $|\arg (z)|=3 \pi / 4$ as in 
Example 4 separating these regimes. This setting holds for a large portion of cases in which $X \in \mathfrak{D}\left(-\mathcal{G}_{\alpha}\right)$. The need for $\beta$ to be strictly less than 1 in the condition (5.4) is illustrated in Example 10 of $\S 7.7$ which considers $X$ as Pareto and also the distribution of $e^{-X}$ when $X$ is Pareto.

\section{Lattice Distributions}

There is no loss in generality in stating our results for distributions on the integer lattice rather than on general lattices. Doing so also serves to avoid the more complicated saddlepoint expressions which occur on general lattices as given in Butler (2007, §2.4.4)

Let $X$ have a lattice distribution on $\{\ldots,-1,0,1, \ldots\}$, with mass function $p(n)$, survival function $S(n)=P(X \geq n)$, and MGF $\mathcal{M}(z)$ defined on $\mathcal{S}_{\mathbb{C}}=\{z \in \mathbb{C}: a \leqq \operatorname{Re}(z)<$ b\} with $-\infty \leq a \leq 0<b \leq \infty$. Let its probability generating function (PGF) $\mathcal{P}(z)$ be defined on the disk $\mathcal{D}=\left\{z \in \mathbb{C}: r_{a} \leqq|z|<r\right\}$ where $r=e^{b}$. The saddlepoint mass function is $\hat{p}(n)=\hat{f}(n)$ with $\hat{f}$ as in (1.2) but restricted to integer-valued $n$.

In the integer-valued setting, $\mathfrak{D}\left(\mathcal{G}_{\alpha}\right)$ and $\mathfrak{D}(\mathcal{N})$ are the only relevant domains of weak convergence of the standardized titled variable $Z_{s}$. Were $X \in \mathfrak{D}\left(-\mathcal{G}_{\alpha}\right)$, then the support of $X$ would be bounded above and the upper boundary of support $t_{U}$ would be approached in finite steps with $\hat{p}\left(t_{U}\right)$ undefined.

Theorem 5. (Limiting saddlepoint mass function ratios). Suppose $b \notin \mathcal{S}$ and either $X \in \mathfrak{D}\left(\mathcal{G}_{\alpha}\right)$ or $X \in \mathfrak{D}(\mathcal{N})$. If there exists a dominating function $D(y)$ with finite integral on $(-\infty, \infty)$, as in (3.2), then

$$
\lim _{n \rightarrow \infty} \frac{p(n)}{\hat{p}(n)}=\left\{\begin{array}{cll}
\hat{\Gamma}(\alpha) / \Gamma(\alpha) & \text { if } & X \in \mathfrak{D}\left(\mathcal{G}_{\alpha}\right) \\
1 & \text { if } & X \in \mathfrak{D}(\mathcal{N}) .
\end{array}\right.
$$

To deal with more difficult cases, weaker conditions than (3.2) are now given which ensure the limits in Theorem 5. By analogy with the continuous case, we assume that the PGF $\mathcal{P}$ has the form $\mathcal{P}(z)=(r-z)^{-\alpha} G(z)$ for some $\alpha>0$. The corresponding MGF is given by

$$
\mathcal{M}(z)=\mathcal{P}\left(e^{z}\right)=\left(e^{b}-e^{z}\right)^{-\alpha} G\left(e^{z}\right) .
$$

The following condition will be required for some $b^{-} \in(0, b)$ : for some $\varepsilon>0$, the function $G\left(e^{z}\right)$ in (6.1) satisfies

$$
\sup _{s \in\left[b^{-}, b\right)}\left|\frac{1}{\sigma_{s}} \frac{G^{\prime}\left(e^{s+i y / \sigma_{s}}\right)}{G\left(e^{s}\right)} 1_{\left\{y:|y|<\pi \sigma_{s}\right\}}\right| \leq c(1+|y|)^{\alpha-1-\varepsilon}
$$

for all $y \in \mathbb{R}$, where $G^{\prime}$ is the complex derivative of $G$ and $c$ is a constant. 
Note that, due to the indicator function $1_{\{\cdot\}}$, the left-hand side of (6.2) is only non-zero when $\sigma_{s}>|y| / \pi$.

Theorem 6. $\left(X \in \mathfrak{D}\left(\mathcal{G}_{\alpha}\right)\right)$. Suppose $X$ is an integer-valued random variable which has moment generating function $\mathcal{M}$ of the form (6.1) and assume $X \in \mathfrak{D}\left(\mathcal{G}_{\alpha}\right)$. If, in addition, $G\left(e^{z}\right)$ satisfies condition (6.2) for some $\varepsilon>0$, then

$$
\lim _{n \rightarrow \infty} \frac{p(n)}{\hat{p}(n)}=\frac{\hat{\Gamma}(\alpha)}{\Gamma(\alpha)}
$$

The Darboux conditions stated in Corollary 6 below were used in Butler $(2017, \S 4)$ to develop expansions for $p(n)$. The next result asserts that the so-called Darboux conditions imply condition (6.2), and therefore they suffice for guaranteeing the saddlepoint limit in (6.3).

Corollary 6. (Darboux conditions). Suppose $X$ has non-negative and integer-valued support, with $\mathcal{P}$ satisfying Darboux conditions, i.e. $\mathcal{P}$ has the form

$$
\mathcal{P}(z)=(r-z)^{-\alpha} G(z), \quad \alpha>0, \quad r>1,
$$

and $G(z)$ is analytic on $\{z \in \mathbb{C}:|z| \leq r\}$ with $G(r) \neq 0$. Then (6.2) holds and therefore the saddlepoint limit in (6.3) holds.

\subsection{Saddlepoint survival approximations}

In the case of integer-valued $X$, we consider four approximations for $S(n)$ denoted as $\hat{S}_{1}(n), \ldots, \hat{S}_{4}(n)$ which were originally introduced in Daniels $(1987, \S 6)$ and further described in Butler $(2007, \S 1.2 .3)$. The value of $\lim _{n \rightarrow \infty} S(n) / \hat{S}_{j}(n)$ depends upon the particular form for $\hat{S}_{j}(n)$, i.e. whether it is continuity-corrected or not and how it is corrected. All four approximations take the form in (1.3) as in the continuous setting but use different values for $\hat{w}$ and $\hat{u}$ in (1.1) and sometimes different saddlepoints $\hat{s}$, which we denote by $\hat{w}_{j}, \hat{u}_{j}$, and $\hat{s}_{j}$ for $j=1,2,3,4$. Table 1 provides these values where $\hat{w}$, $\hat{u}$, and $\hat{s}$ are those values that would be used in the continuous formula with $t=n$ and saddlepoint $\hat{s}$ solving $\mathcal{K}^{\prime}(\hat{s})=n$. Approximation 1 continuity-corrects by using $1-e^{-\hat{s}}$ in place of $\hat{s}$ for $\hat{u}_{1}$. Approximations 2 and 3 use $n^{-}=n-1 / 2$ in place of $n$ and use continuity-corrected saddlepoint $\hat{s}^{-}$solving $\mathcal{K}^{\prime}\left(\hat{s}^{-}\right)=n^{-}$. Approximation 4 does not use continuity correction. Both theory (Daniels, 1987, §6) and computation (Butler, 2007, $\S \S 1.2 .3-1.2 .6)$ support approximations 1 and 2 as the most accurate. 


\begin{tabular}{clll}
\hline \multicolumn{1}{c}{$j$} & \multicolumn{1}{c}{$\hat{w}_{j}$} & \multicolumn{1}{c}{$\hat{u}_{j}$} & \multicolumn{1}{c}{$\hat{s}_{j}$} \\
\hline$\hat{S}_{1}(n)$ & $\hat{w}_{1}=\hat{w}$ & $\hat{u}_{1}=\left(1-e^{-\hat{s}}\right) \sqrt{\mathcal{K}^{\prime \prime}(\hat{s})}$ & $\hat{s}_{1}=\hat{s}$ \\
$\hat{S}_{2}(n)$ & $\hat{w}_{2}=\operatorname{sgn}\left(\hat{s}^{-}\right) \sqrt{2\left\{\hat{s}^{-} n^{-}-\mathcal{K}\left(\hat{s}^{-}\right)\right\}}$ & $\hat{u}_{2}=2 \sinh \left(\hat{s}^{-} / 2\right) \sqrt{\mathcal{K}^{\prime \prime}\left(\hat{s}^{-}\right)}$ & $\hat{s}_{2}=\hat{s}^{-}$ \\
$\hat{S}_{3}(n)$ & $\hat{w}_{3}=\hat{w}_{2}$ & $\hat{u}_{3}=\hat{s}^{-} \sqrt{\mathcal{K}^{\prime \prime}\left(\hat{s}^{-}\right)}$ & $\hat{s}_{3}=\hat{s}^{-}$ \\
$\hat{S}_{4}(n)$ & $\hat{w}_{4}=\hat{w}$ & $\hat{u}_{4}=\hat{u}$ & $\hat{s}_{4}=\hat{s}$ \\
\hline
\end{tabular}

Table 1. Entries into the Lugannani and Rice (1980) formula in (1.3) for computing the four saddlepoint survival approximations for $S(n)$.

Some special subcases arise in the lattice setting that do not occur in the continuous setting; one such subcase will be addressed and the other is excluded from the statement of results. These subcases occur when $X \in \mathfrak{D}(\mathcal{N}), b=\infty$, and $\mathcal{K}^{\prime \prime}(s) \nrightarrow \infty$ as $s \rightarrow \infty$. The two subcases below exhaust possibilities for this setting as it is not possible for $\mathcal{K}^{\prime \prime}(s) \rightarrow 0$ as $s \rightarrow b$ since the limiting distribution would be degenerate and this would violate weak convergence.

In the very light tails case, $X \in \mathfrak{D}(\mathcal{N}), b=\infty$, and

$$
\mathcal{K}^{\prime \prime}(s) \rightarrow \kappa_{\infty}<\infty \quad \text { as } \quad s \rightarrow \infty
$$

while in the divergent case, $X \in \mathfrak{D}(\mathcal{N}), b=\infty$, and

$$
\mathcal{K}^{\prime \prime}(s) \text { diverges with } \quad \liminf _{s \rightarrow \infty} \mathcal{K}^{\prime \prime}(s)>0 \quad \text { and } \quad \limsup _{s \rightarrow \infty} \mathcal{K}^{\prime \prime}(s)<\infty .
$$

Our results do not apply to the divergent variance case (6.5). The discretized normal in Example 12 of $\S 7.7$ provides a context within which both cases (6.4) and (6.5) can occur.

Theorem 7. (Limiting saddlepoint survival ratios). Suppose $b \notin \mathcal{S}$ and the limit

$$
\lim _{n \rightarrow \infty} p(n) / \hat{p}(n)=\lambda \in(0, \infty)
$$

exists as in Theorem 5 or 6 or Corollary 6. Then the four saddlepoint survival function approximations have the following limiting ratios.

(a) If $X \in \mathfrak{D}\left(\mathcal{G}_{\alpha}\right)$,

$$
\lim _{n \rightarrow \infty} \frac{S(n)}{\hat{S}_{j}(n)}=\frac{\hat{\Gamma}(\alpha)}{\Gamma(\alpha)} \times\left\{\begin{array}{ccc}
1 & \text { if } & j=1,2 \\
b /\{2 \sinh (b / 2\} & \text { if } & j=3 \\
b /\left(1-e^{-b}\right) & \text { if } & j=4 .
\end{array}\right.
$$


(b) If $X \in \mathfrak{D}(\mathcal{N})$ but neither (6.4) nor (6.5) holds, and $\hat{u}_{j} / \hat{w}_{j}^{3} \rightarrow 0$ as $\hat{s} \uparrow b$ for $j=1,2,3,4$, then the limiting value for $S(n) / \hat{S}_{j}(n)$ is dependent on whether $b<\infty$ or $b=\infty$, with

$$
\lim _{n \rightarrow \infty} \frac{S(n)}{\hat{S}_{j}(n)}=\left\{\begin{array}{cccc}
1 & \text { if } & j=1,2 & \\
b /\{2 \sinh (b / 2)\} & \text { if } & j=3 & b<\infty \\
b /\left(1-e^{-b}\right) & \text { if } & j=4 & \\
1 & \text { if } & j=1,2 & b=\infty . \\
0 & \text { if } & j=3 & \\
\infty & \text { if } & j=4 &
\end{array}\right.
$$

(c) If $X$ satisfies (6.4) and $\hat{u}_{j} / \hat{w}_{j}^{3} \rightarrow 0$ as $\hat{s} \uparrow b=\infty$ for $j=1,2,3,4$, then the limits are as in the $b=\infty$ case of (6.7) for $j=1,3$, and 4 but for $j=2$,

$$
\lim _{n \rightarrow \infty} \frac{S(n)}{\hat{S}_{2}(n)}=\eta^{-1 / 4}=\exp \left\{-1 /\left(8 \kappa_{\infty}\right)\right\}
$$

where $\kappa_{\infty}$ is given in (6.4).

The first two approximations are asymptotically best since $\hat{\Gamma}(\alpha) / \Gamma(\alpha)<1$ for all $\alpha>0$ and factor $b /(2 \sinh (b / 2)<1$ for $b>0$. Note that there are no conclusions drawn about limiting ratios when $X$ satisfies (6.5) since our method of proof does not cover this situation.

Example 5. (Negative Binomial $(m, p)$ ). Suppose $X$ counts the number of failures before the $m$ th success with $p$ as the probability of success. Then $X \in \mathfrak{D}\left(\mathcal{G}_{m}\right)$ and the dominating function condition (3.2) holds for $m>1$. To see this, note $e^{b}=1 / q$ and

$$
\mathcal{M}(s)=\left(\frac{p}{q}\right)^{m}\left(\frac{1}{q}-e^{s}\right)^{-m}=\left(\frac{p}{q}\right)^{m} A(s)^{-m}(b-s)^{-m}
$$

where $A(z):=\left(e^{b}-e^{z}\right) /(b-z)$ is an entire function with $A(b)=e^{b}$ and no zeros on $\{z \in \mathbb{C}:|\operatorname{Im}(z)| \leq \pi\}$. Then

$$
\begin{aligned}
\sup _{s \in\left[b^{-}, b\right)}\left|\frac{\mathcal{M}\left(s+i y / \sigma_{s}\right)}{\mathcal{M}(s)}\right| & \leq \sup _{s \in\left[b^{-}, b\right)}\left|\frac{A(b)^{+}}{A\left(s+i y / \sigma_{s}\right)}\right|^{m}\left(1+\frac{y^{2}}{\alpha^{+}}\right)^{-m / 2} \\
& \leq \frac{\left\{A(b)^{+}\right\}^{m}}{I^{m}}\left(1+\frac{y^{2}}{\alpha^{+}}\right)^{-m / 2}
\end{aligned}
$$


where $\alpha^{+}=\alpha+\varepsilon$ for some $\varepsilon>0$, and

$$
I=\inf _{s \in\left[b^{-}, b\right]} \inf _{|y| \leq \pi \sigma_{s}}\left|A\left(s+i y / \sigma_{s}\right)\right|=\inf _{s \in\left[b^{-}, b\right]} \inf _{\left|y_{1}\right| \leq \pi}\left|A\left(s+i y_{1}\right)\right|>0 .
$$

The example demonstrates how to show that (3.2) holds and reveals that this is true quite generally when $X \in \mathfrak{D}\left(\mathcal{G}_{\alpha}\right)$ for simpler cases with $\alpha>1$.

Consider a Negative Binomial $(2,0.2)$ example. For the mass function ratio and for $S(n) / \hat{S}_{j}(n)$ for $j=1$ and 2 , the limiting ratio is $\hat{\Gamma}(2)=0.95950$. Pushing the limits of computation in Maple, $S(9000)=1.162 \times 10^{-869}$ and $p(9000) / \hat{p}(9000)=0.95929$. For $\hat{S}_{1}$ and $\hat{S}_{2}$ the survival ratios at 9000 are 0.9639 and 0.9645 respectively. These and other computations suggest that the convergence of $p(n) / \hat{p}(n)$ is faster than for survival ratios. The limits for survival ratios with $j=3$ and 4 should be 0.9575 and 1.071 respectively while their values at 9000 are 0.9625 and 1.076 respectively.

Additional examples are in $\S 7.7$ of Supplementary Materials such as Example 11 which considers $X \sim$ Poisson (1) for which $X \in \mathfrak{D}(\mathcal{N})$. Also Example 12 revisits a discretized normal mass function considered in Balkema et al. (1999a) to show weak convergence of the standardized tilted distribution does not occur. However, subsequences can converge and, depending on the construction, lead to weak limits $\mathfrak{D}(\mathcal{N}), \mathfrak{D}\left(\mathcal{G}_{\alpha}\right)$, or $\mathfrak{D}\left(-\mathcal{G}_{\alpha}\right)$ with (6.4) holding if $\mathfrak{D}(\mathcal{N})$.

For a significance test based on $X$ which rejects for large $X$, the mid- $p$-value is the value $S^{-}(n)=S(n)-p(n) / 2$ when $X=n$ is observed. For a discussion on why mid- $p$ values are preferred to $p$-values, see Agresti (1992) and Butler (2007, §6.1.4). Limiting relative errors for various saddlepoint approximations of this mid-p-value are given in $\S 7.5$ of Supplementary Materials.

\subsection{Saddlepoint error ratios with no limit}

Example 7 in $\S 7.7$ of Supplementary Materials considers a logarithmic series mass function and convolutions this mass function with itself for which $r>1$ is a logarithmic branch point. It provides a class of lattice distributions for which saddlepoint relative errors are not ultimately bounded.

\section{Acknowledgments}

Research of R.W. Butler has been supported by the National Science Foundation under grant DMS-1104474. We are grateful to two referees and an Associate Editor for suggestions which have improved the presentation of this work. 


\section{References}

[1] Abramowitz, M., and Stegun, I.A. (1972). Handbook of Mathematical Functions, 9th ed., New York: Dover.

[2] Agresti, A. (1990). A survey of exact inference for contingency tables. Statistical Science 7, 131-153.

[3] Balkema, A.A., Klüppelberg, C., and Resnick, S.I. (1999a). Limit laws for exponential families. Bernoulli 5, 951-968.

[4] Balkema, A.A., Klüppelberg, C., and Resnick, S.I. (1999b). Domains of attraction for exponential families and asymptotic behaviour of Laplace transforms. Unpublished Research Report.

[5] Balkema, A.A., Klüppelberg, C., and Resnick, S.I. (2003). Domains of attraction for exponential families. Stoch. Proc. Appl. 107, 83-103.

[6] Barndorff-Nielsen, O.E. (1978). Information and Exponential Families in Statistical Theory. Chichester, Wiley.

[7] Barndorff-Nielsen, O. E. (1990). Approximate interval probabilities. J. Royal Statist. Soc. B 52, 485-496.

[8] Barndorff-Nielsen, O.E. and Klüppelberg, C. (1992). A note on the tail accuracy of the univariate saddlepoint approximation. Ann. Tolouse (Sér 6). 1, 5-14.

[9] Barndorff-Nielsen, O.E. and Klüppelberg, C. (1999). Tail exactness of multivariate saddlepoint approximations. Scand. J. Statist. 26, 253-264.

[10] Bingham, N.H., Goldie, C.M. and Teugels, J.L. (1987). Regular Variation. Cambridge: Cambridge University Press.

[11] Bleistein, N. and Handelsman, R.A. (1975). Asymptotic Expansions of Integrals. New York, Dover.

[12] Booth, J.G. (1994). A note on the accuracy of two saddlepoint tail approximations. Proc. Amer. Statist. Assoc.-Phys. 83 Engr. Sci.

[13] Butler, R.W. (2000). Reliabilities for feedback systems and their saddlepoint approximation. Statist. Sci. 15 279-298.

[14] Butler, R.W. (2007). Saddlepoint Approximations with Applications. Cambridge: Cambridge University Press.

[15] Butler, R.W. (2017). Asymptotic expansions and hazard rates for compound and first-passage distributions. Bernoulli, 23, 3508-3536.

[16] Butler, R.W. (2018). Asymptotic expansions and saddlepoint approximations for first-passage distributions in semi-Markov processes. In preparation.

[17] Copson, E.T. (1965). Asymptotic Expansions. Cambridge: Cambridge University Press.

[18] Daniels, H.E. (1954). Saddlepoint approximations in statistics. Ann. Math. Statist., 25, 631-650.

[19] Daniels, H.E. (1980). Exact saddlepoint approximations. Biometrika, 67, 59-63. 
[20] Daniels, H.E. (1987). Tail probability approximations. Int. Statist. Rev., 55, 37-48.

[21] Daniels, H.E. (1991). A class of nearly exact saddlepoint approximations. The Art of Statistical Science (ed. K. Mardia) New York: Wiley.

[22] Doetsch, G. (1974). Introduction to the Theory and Application of the Laplace Transform. New York: Springer.

[23] Feller, W. (1971). An Introduction to Probability Theory and Its Applications, Vol. II. 2nd ed. New York: Wiley.

[24] Flajolet, P. and Odlyzko, A. (1990). Singularity analysis of generating functions. SIAM J. Disc. Math. 3, 216-240.

[25] Henrici, P. (1977). Applied and Computational Complex Analysis, vol. 2. New York: Wiley.

[26] Huang, X.-C. (1988). A discrete L'Hopital's rule. College Math. J. 19, 321-329.

[27] Jensen, J.L. (1988). Uniform saddlepoint approximations. Adv. Appl. Probab. 20 622-634.

[28] Jensen, J.L. (1991a). Uniform saddlepoint approximations and log-concave densities. J. Roy. Statist. Soc. B 53 157-172.

[29] Jensen, J.L. (1991b). Saddlepoint approximations to the distribution of the total claim amount in some recent risk models. Scand. Actuarial J. 1991 154-168.

[30] Jensen, J.L. (1995). Saddlepoint Approximations. Oxford: Clarendon Press.

[31] Jorgensen, B. (1980). Statistical Properties of the Generalized Inverse Gaussian Distribution. Lecture Notes in Statistics, Vol 9. New York: Springer-Verlag.

[32] Korevaar, J. (2004). Tauberian Theory: A Century of Developments. Berlin, Springer.

[33] Lugannani, R. and Rice, S. (1980) Saddlepoint approximations for the distribution of the sum of independent random variables. Adv. Appl. Probab., 12, 475-490.

[34] Lukacs, E. (1964). Inversion formulae for characteristic functions of absolutely continuous distributions. Amer. Math. Monthly 71 44-47.

[35] McCullagh, P. (1994). Does the moment-generating function characterize a distribution? Amer. Statist., 48, 208.

[36] NIST Digital Library of Mathematical Functions. http://dlmf.nist.gov/, Release 1.0.8 of 2014-04-25.

[37] Paige, R.L., Chapman, P.L. and Butler, R.W. (2011). Small sample LD-50 confidence intervals using saddlepoint approximations. Journal of the American Statistical Association, 106, 334-344.

[38] Vuilleumier, M. (1976). Slowly varying functions in the complex plane. Trans. Am. Math. Soc. 218, 343-348.

[39] Widder, D.V. (2010). The Laplace Transform. Republication of 1941 Edition. New York, Dover. 